\title{
Diagnosis of Neurosyphilis through Treponemal methods based on Hemagglutination in Cerebrospinal Fluid in PLWHIV and without HIV: Systematic review
}

Beatriz Azevedo ( $\nabla$ beatrizpereiradeazevedo@gmail.com )

Research article

Keywords: Neurosyphilis, CSF, Diagnosis, TPPA, TPHA

Posted Date: January 17th, 2022

DOI: https://doi.org/10.21203/rs.3.rs-1265393/v1

License: (c) (1) This work is licensed under a Creative Commons Attribution 4.0 International License. Read Full License 


\section{Abstract}

Neurosyphilis (NS) is the severe form of syphilis caused by the invasion of Treponema Pallidum subspecies pallidum in the Central Nervous System (CNS). The laboratory diagnosis is based on the analyses of Cerebrospinal Fluid (CSF) with the VDRL, considered "gold standard", despite recent studies reporting low sensitivity (40-70\%). The treponemal tests based on hemagglutination was performed for use in serum and plasma with great diagnostic accuracy. PURPOSE: This systematic review aims to analyze original studies with treponemal tests based hemagglutination on CSF in order to verify the diagnostic accuracy of these tests in the diagnosis of NS. METHODS: A search was performed in the electronic databases: EMBASE, Scielo, MEDLINE/PUBMED, LILACS, Cochrane Library, Trials and "grey literature" with the following DECS contained in the title or abstract, in English, Portuguese and Spanish: "Neurosyphilis" and "Cerebrospinal fluid" and "diagnosis" and "TPPA" or "TPHA" from 2010 to June 2021. RESULTS: 317 articles were identificated for reading the title and abstract and 27 articles were selected for full reading. Studies have shown that treponemal tests based on hemagglutination are useful when the CSF-VDRL is non-reactive and there are neurological symptoms. CONCLUSION:The sensitivity of CSFTPPA or CSF-TPHA ranges from $75-85 \%$ and specificity ranges from $88.9 \%$ to $100 \%$ with titers $\geq 1: 640$ in TPPA and $\geq 1: 80$ in TPHA for the diagnosis of NS while when titers $<1: 640$ are used, the sensitivity and specificity tend to decrease. There was no difference in the accuracy of the tests between PLWHIV and without HIV.

\section{Introduction}

Neurosyphilis (NS) is a form of presentation of syphilis caused by the spirochete Treponema Pallidum subspecies pallidum (Tp) that happens when the bacterium invades the Central Nervous System (CNS). Transmission occurs through contact with the mucosa and the neurological involvement is due to the ability of Tp to cross vascular barriers [1]. The clinical manifestations of NS can occur at any stage of syphilis, but in some cases the disease is asymptomatic [2].

Since 2010, syphilis has become a notifiable disease in Brazil. According to the Epidemiological report of the Brazilian Ministry of Health (2021), there was an increase in the syphilis detection rate until 2018, with 76.8 cases per 100.000 inhabitants. In 2018 and 2019 there was a reduction in the number of notifications, reaching 54.5 cases per 100.000 population, which may have been caused by underreporting of cases due to the greater mobility of health professionals in combating the COVID-19 pandemic [3].

The HIV-Syphilis coinfection alters the clinical management and therapeutic response of both infections. HIV can alter the lesions, diagnosis and response to syphilis treatment as well as accelerate the progression. There are reports of overlapping of primary and secondary phases in co-infected patients [4]. It's important to highlight that soon after the emergence of HIV, increasing reports of NS were observed among patients infected with the HIV virus, even among those who had received adequate therapy with penicillin [5]. 
Otherwise syphilis can increase the risk of HIV transmission and infection through transient immunosuppression and a weakening of the host's response to the virus. In addition, syphilitic lesions are well vascularized and exposed, which facilitates the entry of HIV into the bloodstream [6].

The diagnosis of NS is performed through clinical aspects and the analysis of Cerebrospinal Fluid (CSF) with a combination of pleocytosis (cells $\geq 5 / \mu \mathrm{L}$ ), blood barrier dysfunction and the reactivity of the Venereal Disease Research Laboratory (VDRL) which is considered the "gold standard" for the diagnosis of NS [7]. Studies with the CSF-VDRL report a high specificity of the test, however, a low sensitivity (30$70 \%$, which makes the diagnosis of NS a constant challenge, especially in asymptomatic patients [8]

The Fluorescent Treponemal Antibody Absorption Test (FTA-Abs) in the CSF is a treponemal test with a lower specificity but with high sensitivity compared to the CSF-VDRL [9]. The Center for Disease Control and Prevention (CDC) considers FTA-Abs as a confirmatory test in the diagnosis of NS so NS is unlikely with a FTA-Abs test in non-reactive CSF [10-11].

The Treponema Pallidum Hemagglutination Test (TPHA) and the Treponema pallidum particle agglutination assay (TPPA) are treponemal tests similar to FTA-Abs, using serial dilutions and are based on a hemagglutination reaction in the presence of antibody against $T p$, which enables an reading the result with the naked eye, unlike the FTA-Abs which requires a fluorescence microscope [12].

The sensitivity of treponemal tests in the CSF is greater than $90 \%$ when the NS criterion consists of CSFVDRL reactive, and lower when the NS criterion is based on neurological symptoms [13]. Marra et al. (2017) in their study of CSF-TPPA demonstrated a sensitivity of $77.5 \%$ based on the symptomatic criterion and $95 \%$ when based on the criterion of reactive CSF-VDRL. The specificity with TPPA titers $\geq$ $1: 640$ was $93.9 \%$ with the definition of NS based on the CSF-VDRL and $85.2 \%$ if using symptomatic criteria. The study also reports a possible increase of $21.3 \%$ in the diagnosis of NS with the combination of CSF-VDRL and CSF-TPPA with titers $\geq 1: 640$ [14].

This systematic review aims to analyze original studies with CSF hemagglutination-based treponemal tests in order to verify the diagnostic accuracy of these tests in the diagnosis of NS.

\section{Materials And Methods}

For this survey, an electronic search was carried out in the following databases: EMBASE, Scielo, MEDLINE/PUBMED, LILACS, Cochrane Library with the descriptors in English, Portuguese and Spanish: "Neurosyphilis" and "Cerebrospinal Fluid" and "diagnosis" and "TPPA" or "TPHA". The same descriptors were also searched in Trials databases (Clinical Trials, ICTRP, as well as in the Brazilian databases of "grey literature" in the Catalog of Theses and Dissertations in the database of the Ministry of Science and Technology. The search was limited to the period of January 2010 to June 2021, with the purpose of selecting recent studies on the use of these treponemal tests based on hemagglutination in cerebrospinal fluid in the diagnosis of NS. 


\section{Inclusion/Exclusion Criteria}

The inclusion criteria used were: studies involving treponemal tests that use the hemagglutination method as a basis, more specifically TPHA and TPPA, in PLHIV and without HIV infection related to the diagnosis of NS, which should contain: study site, number of the sample, characteristics of the population in relation to HIV, type of hemagglutination-based treponemal test (TPPA or TPHA), test titulation and measures of diagnostic accuracy.

\section{Results/ Discussion}

A total of 317 articles were identified in the following databases: 18 in Scielo, 170 in Pubmed, 0 in LILACS, 106 in Embase, 12 in the Cochrane Library and 11 in the Catalog of Theses and Dissertations in the database of the Ministry of Science and Technology. 27 articles were selected for full reading, and 290 were excluded after reading the title and abstract (Figure 1).

Among the articles excluded after reading the title and abstract, 68 were case studies, 6 review studies, 4 observational studies, 21 addressed other pathogens, 16 associated syphilis with other pathogens, 6 addressed the treatment of syphilis, 3 reported other techniques used in the CSF and 167 articles addressed only serological techniques.

After the complete reading of the 27 selected articles, 11 articles were included and 16 were excluded for not following the inclusion criteria, such as: four studies use the TPHA as an inclusion criteria, two report NS as a neurological finding, one uses other techniques of diagnosis, one is a review article, two due to inability to access the full article, one article published in 1988 and five duplicate articles.

Figure 1: Flowchart of electronic search in databases.

(Insert here Figure 1)

It was observed that the selected articles included a total of 2,168 patients who met the inclusion criteria proposed in this review.

Regarding the groups of patients living with HIV (PLHIV), only seven of the selected studies studied the PLHIV and non-HIV group, two studies analyzed only PLHIV and six studies did not include people living with HIV.

\section{Sensitivity and specificity}

When analyzing the selected articles, a total of 3.096 patients were observed, being 1.247 PLWHIV and 1.878 without HIV. The studies involving patients from both groups did not differentiate the sensitivity and specificity of the test according to each group except for two studies $[14,15]$ that reported no difference in the sensitivity and specificity of the test between PLWHIV and those without HIV. 
Only seven of the 11 studies reported the sensitivity and specificity found in the titulation of the test used for the diagnosis and/or follow-up of the treatment of NS. According to the analysis of selected articles, four articles report a sensitivity of CSF-TPPA or CSF-TPHA of 75-85\% and five articles with a specificity of $88.9 \%$ to $100 \%$, it is important to emphasize that when the titration parameter for NS is $<1: 640$ the specificity and sensitivity of the test tend to decrease. In controversy to what is reported in the study by Marra et al. (2021), where a sensitivity of $12-48 \%$ and specificity of $89-96 \%$ were found with titers $\geq 1: 640$ in TPPA in the CSF.

A study in PLWHIV uses the division into two groups: symptomatic NS and asymptomatic NS and reports specificity and sensitivity of each. The TPPA test in the symptomatic NS group had a sensitivity of $72 \%$

and specificity of $52 \%$ and in the asymptomatic NS group, a sensitivity of $50 \%$ and specificity of $47 \%$ [16].

In a study that followed three patients before and after NS treatment, the TPHA titre declined one titer in two patients and two titers in the remaining patient. There was a conversion of CSF-VDRL non-reactivity in two of the three patients [12].

Only five of the seven studies using TPPA reported having followed the manufacturer's guidelines (SERODIA®-TP-PA, Fujirebio Diagnostics Inc), while the TPHA studies did not describe guidelines or manufacturers except for one Cuban study that used the TPHA from the manufacturer CENTIS ${ }^{\circledR}$. In addition, TPHA is not available in all countries, being more commonly found in South American countries. In Brazil we have Imuno-HAIß-TPHA (WAMA Diagnóstica) being commercially used for serum and/or plasma samples.

\section{Diagnostic Screening}

When analyzing the diagnostic screening, it was observed that only two studies didn't include neurological signs and symptoms, all studies included LCR-VDRL reactivity, five determined LCR-RPR reactivity, one in TRUST, one in FTA-Abs $(\mathrm{IgG})$ and one with detection of Tp by the CSF RT-PCR technique.

Indefinite syphilis and patients with serological scarring were considered inclusion criteria only by Salamano et al. (2015). Findings such as pleocytosis and proteinorrhachia were associated in NS cases in 5 studies. All studies involving only PLWHIV considered the serological levels in the VDRL or RPR $\geq 1: 32$ and the T-CD4+ lymphocyte count with a cutoff of $350 \mathrm{cells} / \mathrm{mm} 3$ as diagnostic screening.

\section{Comparison of TPHAVTPPA titers between PLHIV and seronegative groups.}

Among the studies analyzed with TPHA, only Pérez et al. (2013), in their HIV+ and HIV- population, reported the manufacturer (CENTIS $\AA$ ) and its use in the CSF followed the instructions insert for use in serum/plasma, with reactivity titration $\geq 1: 80$.

A wide use of SERODIA-TPPA® (5 of 11 studies) was observed, differing in population data, CSF reactivity titulation and diagnostic accuracy test, summarized in Table 1. 
Table 1: Selected articles relation with titration and diagnostic accuracy data.

(Insert here Table1)

Two studies with SERODIA-TPPA that analyzed the population only without HIV used a titer of $\geq 1: 80$ and their statistical results were in agreement with CSF-VDRL and CSF-TRUST and total percentage of patients with reactive CSF-TPPA (19-20). LI et al. (2021) use the same HIV population with a titer $\geq 1: 320$ and obtain a sensitivity of $84.09 \%$ and a specificity of $88.89 \%$.

Dumaresq and collaborators (2013) performed the study only with PLWHIV, resulting in a sensitivity of $72 \%$ and specificity of $52 \%$ in the symptomatic NS group and $50 \%$ sensitivity and $47 \%$ specificity in the asymptomatic NS group. However, the authors do not describe which cutoff titulation for reactivity, which prevents a better understanding and comparison of the present review [16].

In the study by Guarner et al. (2015), the study population included PLWHIV and without HIV using the SERODIA-TPPA $®$ with a CSF reactivity cutoff of $\geq 1: 640$, resulting in a diagnostic accuracy with a sensitivity of $83.3 \%$ and $100 \%$ specificity [23].

Despite the tests routinely used for the diagnosis, it is necessary to develop and innovate new techniques to improve the diagnostic accuracy of NS. Recently, molecular biology techniques and detection of new markers such as CXCL13 and allelic profiling have been introduced in the diagnosis of NS $[25,26]$.

\section{Conclusion}

Treponemal methods based on hemagglutination in the CSF are targets of research with the aim of improving the laboratory diagnosis of Ns, especially when the VDRL, considered as the "gold standard" for diagnosis, is not reactive, due to its low sensitivity $(40 \%-70 \%)$ in the LCR. [7].

Hemagglutination-based Treponemal tests (TPHA and TPPA) are commercially validated for use in Serum or Plasma and their use in CSF is reported for research purposes, being useful when the VDRL is not reactive and there are neurological symptoms compatible with the NS.

There was no difference between PLWHIV and without HIV in the accuracy of the CSF-TPPA and CSFTPHA tests.

The sensitivity of CSF-TPPA or CSF-TPHA ranges from $75-85 \%$ and specificity from $88.9-100 \%$ with titers $\geq 1: 640$ in TPPA and $\geq 1: 80$ in TPHA for the diagnosis of NS. When titers $<1: 640$ are used, the sensitivity and specificity of the CSF treponemal hemagglutination tests tend to decrease.

\section{Declarations}

a. Funding: The authors declare that no funds, grants, or other support were received during the preparation of this manuscript. 
b. Competing Interests: The authors have no financial, commercial, political, academic and personal conflicts of interest.

c. Data Availability: Not applicable.

d. Code availability: Not applicable.

e. Ethics approval: This is a review study. The Research Ethics Committee (HUGG) has confirmed that no ethical approval is required.

f. Author Contributions: All authors contributed to the conception and design of the study. Material preparation, data collection and analysis were performed by Beatriz Azevedo and Isabelle Rangel. The first draft of the manuscript was written by Beatriz Azevedo and all authors commented on previous versions of the manuscript. All authors read and approved the final manuscript.

g. Consent to participate: Not applicable.

h. Consent for publication: Not applicable.

\section{References}

1.Há Tuan ; Prasanna Tadi ; Laurence Dubensky .Neurosyfilis. StatPearls. Ilha do Tesouro (FL): StatPearls Publishing; 2019-.2019 11 de maio.

2. Marra, C. M. (2015). Neurosyphilis. CONTINUUM: Lifelong Learning in Neurology, 21,1714-1728. doi:10.1212/con.0000000000000250.

3. BRASIL.Ministério da Saúde. Secretaria de Vigilância em Saúde. Boletim Epidemiológico Sífilis 2021. Ministério da Saúde. Secretaria de Vigilância em Saúde - Brasília, 2020 < Available from > https://www.gov.br/saude/pt-br/media/pdf/2021/outubro/14-1/boletim_sifilis-2021_internet.pdf Acess in: November, 2021

4. Zetola NM, Klausner JD. Syphilis and HIV infection: an update. Clin Infect Dis 2007;44:1222-8

5. Berry CD, Hooton TM, Collier AC, Lukehart SA. Neurologic relapse after benzathine penicillin therapy for secondary syphilis in a patient with HIV infection. N Engl J Med. 1987 Jun 18;316(25):1587-9. doi: 10.1056/NEJM198706183162507. PMID: 3587291.

6. Ghanem, K. G. (2010). Evaluation and Management of Syphilis in the HIV-Infected Patient. Current Infectious Disease Reports, 12(2), 140-146. doi:10.1007/s11908-010-0083-6

7. Brasil. Ministério da Saúde. Secretaria de Vigilância em Saúde. Departamento de Vigilância, Prevenção e Controle das Doenças Sexualmente Transmissíveis, Aids e Hepatites Virais. Manual Técnico para Diagnóstico da Sífilis. Brasília: Ministério da Saúde, 2018. 
8. Ho Emily , Clare L Maxwell, Shelia B Dunaway, Sharon K Sahi, Lauren C Tantalo, Sheila A Lukehart, and Christina Marra.Neurosyphilis Increases Human Immunodeficiency Virus (HIV)-associated Central Nervous System Inflammation but Does Not Explain Cognitive Impairment in HIV-infected Individuals With Syphilis.Clin Infect Dis. 2017 Sep 15; 65(6): 943-948.Published online 2017 May 19. doi: $10.1093 / \mathrm{cid} / \mathrm{cix} 473$.

9. Harding AS, Ghanem KG. The performance of cerebrospinal fluid treponemal-specific antibody tests in neurosyphilis: a systematic review. Sex Transm Dis. 2012 Apr;39(4):291-7. doi:

10.1097/OLQ.0b013e31824c0e62. PMID: 22421696.

10. Workowski KA, Berman S. Centers for Disease Control and Prevention (CDC). Sexual Transmitted Disease Treatment Guidelines, 2010. MMWR Recomm Rep 2010; 59:1-110.

11. Kaplan JE, Benson C, Holmes KH, et al. Guidelines for prevention and treatment of opportunistic infections in HIV-infected adults and adolescents: Recommendations from CDC, the National Institutes of Health, and the HIV Medicine Association of the Infectious Diseases Society of America. MMWR Recomm Rep 2009; 58:1, 207; quiz CE1-4.

12. Levchik N, Ponomareva M, Surganova V, Zilberberg N, Kungurov N. Criteria for the diagnosis of neurosyphilis in cerebrospinal fluid: relationships with intrathecal immunoglobulin synthesis and bloodcerebrospinal fluid barrier dysfunction. Sex Transm Dis. 2013 Dec;40(12):917-22. doi:

10.1097/OLQ.0000000000000049. PMID: 24220351.

13. Castro R, Prieto ES, Aguas MJ, Manata MJ, Botas J, Araujo C, Borges F, Aldir I, Exposto FDL. 2006. Evaluation of the Treponema pallidum particle agglutination technique (TP.PA) in the diagnosis of neurosyphilis. J Clin Lab Anal 20:233-238. doi:10.1002/jcla.20147

14. Marra CM, Maxwell CL, Dunaway SB, Sahi SK, Tantalo LC. Cerebrospinal Fluid Treponema pallidum Particle Agglutination Assay for Neurosyphilis Diagnosis. J Clin Microbiol. 2017;55(6):1865-1870. doi:10.1128/JCM.00310-17

15. Marra CM. Alternatives to the Cerebrospinal Fluid Venereal Disease Research Laboratory Test for Neurosyphilis Diagnosis. Sex Transm Dis. 2021 Aug 1;48(8S):S54-S57. doi:

10.1097/OLQ.0000000000001450. PMID: 33967236.

16. Dumaresq, J., Langevin, S., Gagnon, S., Serhir, B., Deligne, B., Tremblay, C., Tsang, R. S., Fortin, C., Coutlée, F., \& Roger, M. (2013). Clinical prediction and diagnosis of neurosyphilis in HIV-infected patients with early Syphilis. Journal of clinical microbiology, 51(12), 40604066. https://doi.org/10.1128/JCM.01989-13

17. Salamano, Ronald et al. Cerebrospinal fluid examination may be useful in diagnosing neurosyphilis in asymptomatic HIV+ patients with syphilis. Arquivos de Neuro-Psiquiatria [online]. 2016, v. 74, n. 2 , pp. 128-132. https://doi.org/10.1590/0004-282X20160016. 
18. Echevarria-Pérez E, Rodríguez-González I, Fernández-Molina C.Value in practice of the cerebrospinal fluid in Treponema pallidum hemagglutination test for confirmation of neurosyphilis. Revista Cubana de Medicina Tropical. 2013; 65(2): 258-263.

19. Chen YY, Zhang YF, Qiu XH, Zhang Q, Chen FY, Liu L, Fan JY, Gao K, Zhu XZ, Zheng WH, Zhang HL, Lin LR, Liu LL, Tong ML, Niu JJ, Yang TC. Clinical and laboratory characteristics in patients suffering from general paresis in the modern era. J Neurol Sci. 2015 Mar 15;350(1-2):79-83. doi:

10.1016/j.jns.2015.02.021. Epub 2015 Feb 17. PMID: 25703276.

20. Gu, W., Yang, Y., Wu, L., Yang, S., \& Ng, L. K. (2013). Comparing the performance characteristics of CSF-TRUST and CSF-VDRL for syphilis: a cross-sectional study. BMJ open, 3(2), e002204. https://doi.org/10.1136/bmjopen-2012-002204

21. Li D, Huang X, Shi M, et al. Diagnostic role of CXCL13 and CSF serology in patients with neurosyphilis. Sexually Transmitted Infections Published Online First: 12 January 2021. doi: 10.1136/sextrans-2020-054778

23. Guarner, J., Jost, H., Pillay, A., Sun, Y., Cox, D., Notenboom, R., \& Workowski, K. (2015). Evaluation of treponemal serum tests performed on cerebrospinal fluid for diagnosis of neurosyphilis. American journal of clinical pathology, 143(4), 479-484. https://doi.org/10.1309/AJCPWSL3G8RXMCQR

24. Kissani N, Nafia S, Zahlane S, Louhab N. Neurosyphilis: a series of 178 cases at the 3rd-level hospital of Marrakesh (Morocco). Eur J Clin Microbiol Infect Dis. 2021 May 3. doi: 10.1007/s10096-021-04253-y.

25. Grillová L, Bawa T, Mikalová L, Gayet-Ageron A, Nieselt K, Strouhal M, Sednaoui P, Ferry T, Cavassini M, Lautenschlager S, Dutly F, Pla-Díaz M, Krützen M, González-Candelas F, Bagheri HC, Šmajs D, Arora N, Bosshard PP. Molecular characterization of Treponema pallidum subsp. pallidum in Switzerland and France with a new multilocus sequence typing scheme. PLoS One. 2018 Jul 30;13(7):e0200773. doi: 10.1371/journal.pone.0200773. PMID: 30059541; PMCID: PMC6066202.

26. CARVALHO, RICARDO DE SOUZA. Diagnostic value of CXCL13 cerebrospinal fluid dosage in the diagnosis of neurosyphilis in patients living with HIV.30/12/2020.Doutorado em Neurologia. Instituição de Ensino: Universidade Federal do Estado do Rio de Janeiro, Rio de Janeiro. Biblioteca Depositária: UNIRIO.

\section{Table 1}

Table 1 is available in the Supplementary Files section.

\section{Figures}




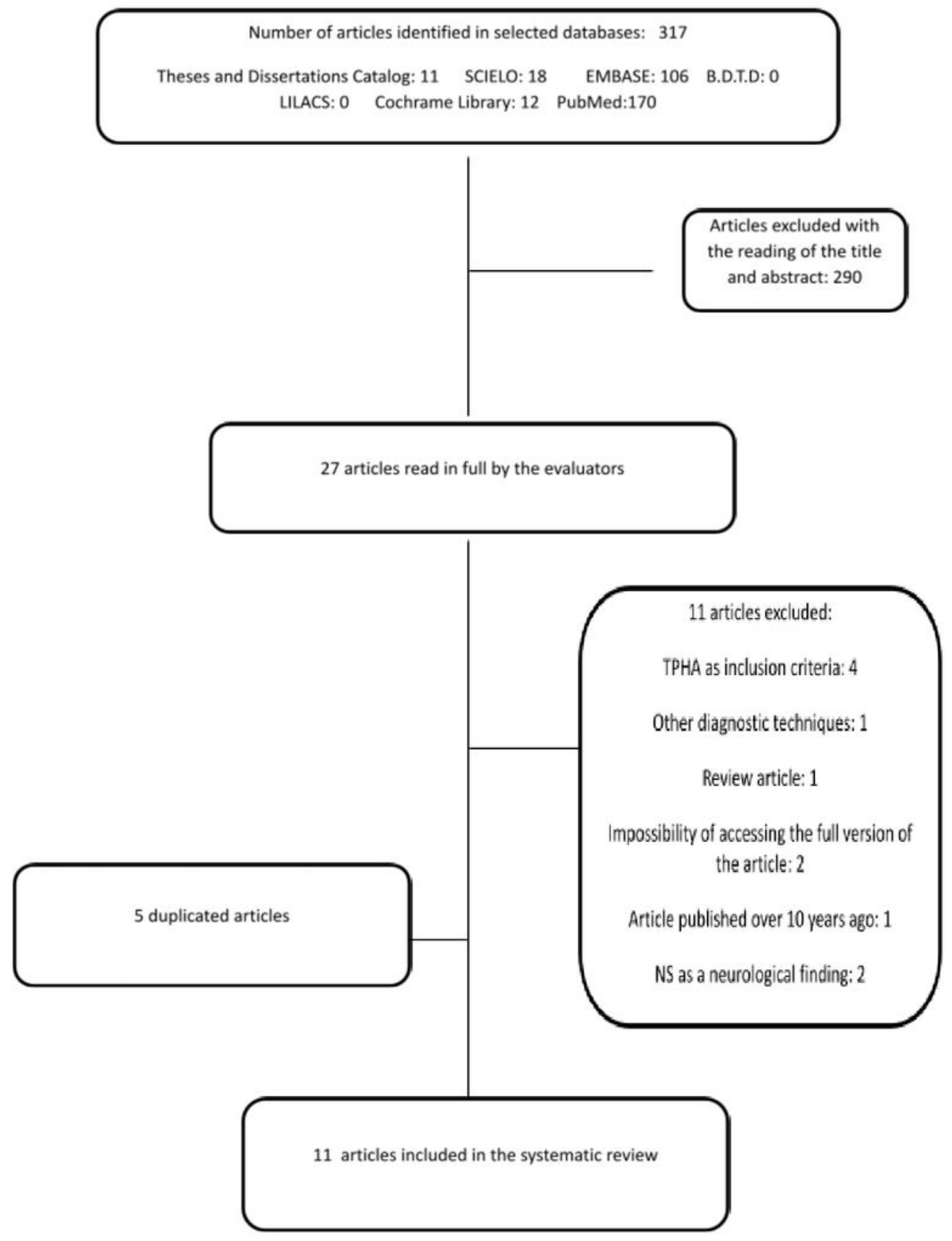

Figure 1

Flowchart of electronic search in databases.

\section{Supplementary Files}

This is a list of supplementary files associated with this preprint. Click to download. 
- PlanilhaReview11.xlsx

Page $11 / 11$ 